\title{
FORMATION AND CONTROL OF COULOMB CRYSTALS IN TRAPPED ION PLASMAS
}

\author{
X.-P. Huang, J. J. Bollinger, W. M. Itano, J. N. Tan, ${ }^{1}$ B. Jelenković, ${ }^{2}$ \\ T. B. Mitchell, and D. J. Wineland \\ Time \& Frequency Division, NIST \\ Boulder, $\mathrm{CO}$ \\ 'Present address: Frequency \& Time Systems, Beverly, MA \\ ${ }^{2}$ On leave from the Institute of Physics, University of Belgrade, Yugoslavia
}

\section{INTRODUCTION}

Trapped non-neutral plasmas consisting of one charged particle species provide an experimental realization of a classical one-component plasma (OCP). ${ }^{\prime}$ In Penning traps, which use static electric and magnetic fields for confinement, trapped plasmas can relax to a global thermal equilibrium which undergoes a rigid-body rotation about the magnetic field axis. ${ }^{2}$ In a frame rotating with the plasma, there arises an induced electric field which takes the place of the field from the uniform neutralizing background in the OCP model. Active control of the rotation frequency prevents plasmas from spinning down under the ambient drag from static field errors and background neutral molecules, and allows variation of the plasma density and shape. ${ }^{2,3}$

With Doppler laser cooling, pure ion plasmas with density $n_{0}$ greater than $10^{8} \mathrm{~cm}^{-3}$ and temperature $T$ less than $5 \mathrm{mK}$ can be routinely obtained, ${ }^{2}$ resulting in a Coulomb coupling parameter $\Gamma \equiv\left(e^{2} / 4 \pi \epsilon_{0} a_{\mathrm{WS}}\right)\left(k_{\mathrm{B}} T\right)^{-1}$ greater than 200 . Here, $e$ is the ion charge and $a_{\mathrm{WS}}$ is the Wigner-Seitz radius defined by $4 \pi a_{\mathrm{WS}}^{3} / 3 \equiv 1 / n_{0}$. A classical, infinite OCP freezes into a bcc lattice at $\Gamma \approx 172 .{ }^{4}$ However, this result does not strictly apply to the trapped plasmas because of the surface effects associated with their finite size. Both simulations ${ }^{5}$ and experiments ${ }^{6}$ show that a structure of concentric shells forms for nearly spherical plasmas with $10^{3}$ to $10^{4}$ ions. For plasmas with $\gtrsim 2 \times 10^{5}$ ions or $\gtrsim 30$ shells, time-averaged Bragg scattering patterns are consistent with bcc crystals (presumably located near the plasma center) ${ }^{7}$ in agreement with a theoretical estimate. ${ }^{8}$ But this measurement can not determine whether the Bragg patterns come from single crystals or polycrystals.

In this report, we demonstrate that azimuthally asymmetric electric fields rotating in the same sense as the plasma can phase-lock the rotation of crystallized plasmas without slip, therefore precisely controlling the plasma rotation frequency, density, and surface shape. ${ }^{9} \mathrm{We}$ synchronize the detection of Bragg-scattered light either with this active rotation control or using the time dependence of the scattered light itself measured by a fast photomultiplier tube. Time-resolved (stroboscopic) Bragg diffraction patterns are obtained, effectively removing 

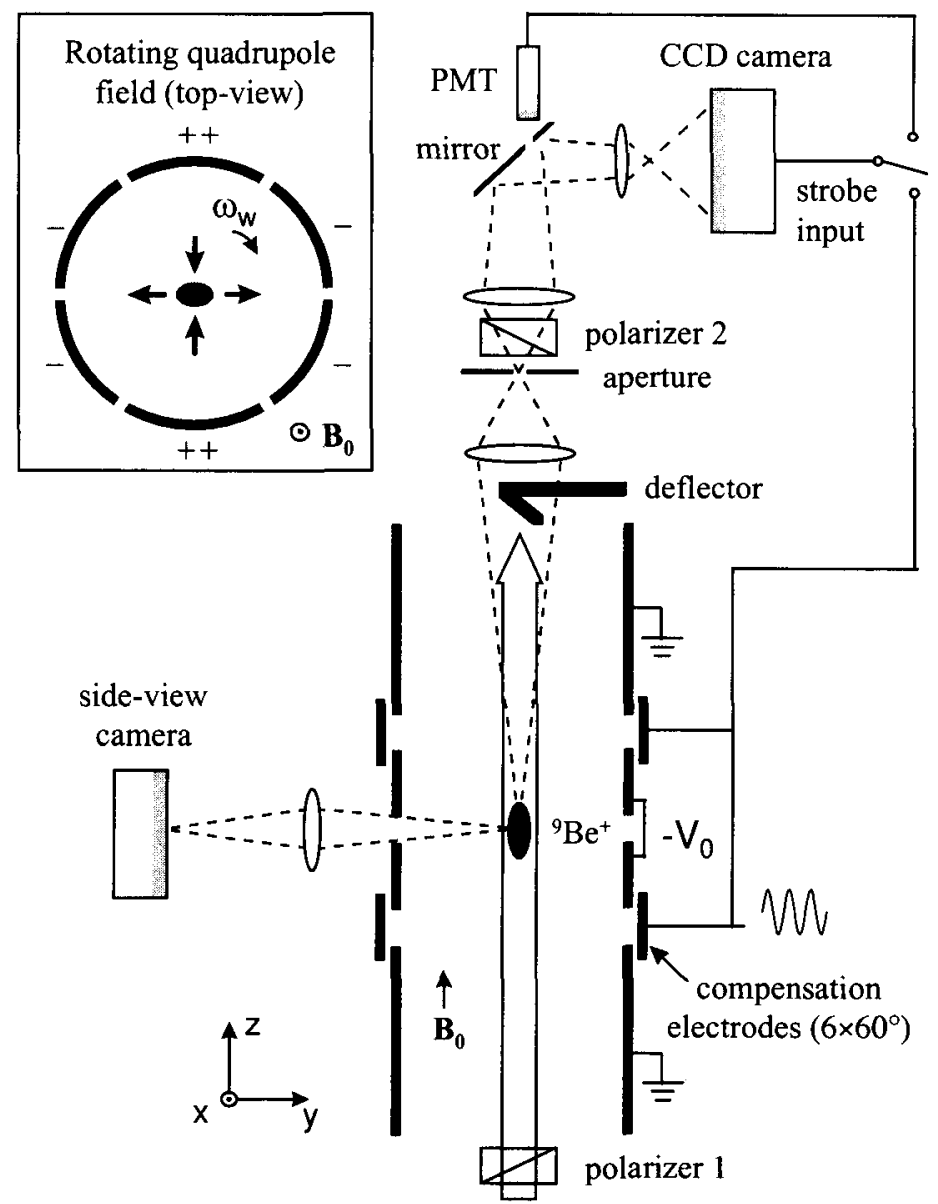

axial cooling

beam $(313 \mathrm{~nm})$

Figure 1. Schematic of the experimental setup.

the plasma rotation. ${ }^{10}$ Patterns from single bcc lattices are observed most of the time in these plasmas, ${ }^{10}$ in agreement with the theoretically predicted bulk structure of a solid onecomponent plasma. ${ }^{4}$ In addition, some preferred crystal orientations with respect to the axial laser beam are also seen. With phase-locked rotation, the lattice and its orientation can be stable for longer than $30 \mathrm{~min}$, compared to an observed $\sim 1$ min lifetime without the active rotation control.

\section{EXPERIMENTAL SETUP}

Figure 1 shows the apparatus and the asymmetric rotating field. The trap consists of a $127 \mathrm{~mm}$ long stack of cylindrical electrodes at room temperature with an inner diameter of $40.6 \mathrm{~mm}$, enclosed in a $10^{-8} \mathrm{~Pa}$ vacuum chamber. An axisymmetric potential $\propto\left[z^{2}-\left(x^{2}+\right.\right.$ $\left.y^{2}\right) / 2$ is generated by biasing the central electrodes to $-V_{0}$, giving axial particle confinement. A uniform magnetic field $B_{0}=4.46 \mathrm{~T}$ from a superconducting magnet is aligned parallel to the trap axis, resulting in global rotation and radial trapping. As shown in the inset, a 

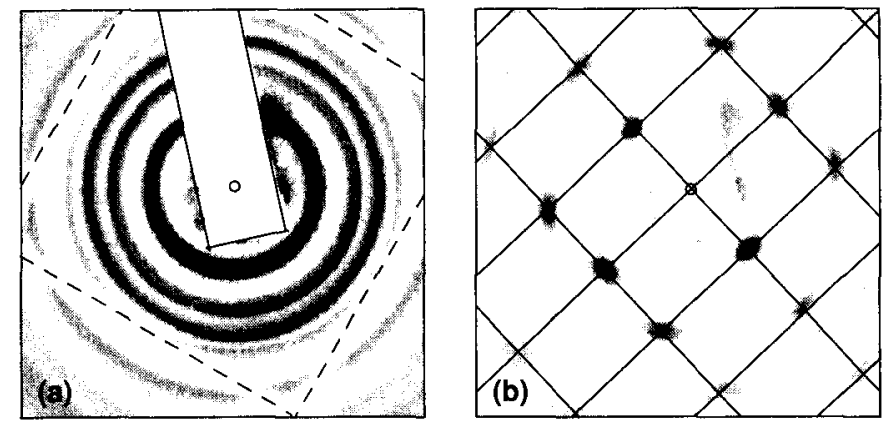

Figure 2. Bragg diffraction from a plasma phase-locked to the rotating field $\left(\omega_{r}=\omega_{w}=2 \pi \times 140 \mathrm{kHz}\right.$, $n_{0}=4.26 \times 10^{8} \mathrm{~cm}^{-3}$ ). (a) $1 \mathrm{~s} \mathrm{time-averaged} \mathrm{pattern.} \mathrm{The} \mathrm{long} \mathrm{rectangular} \mathrm{shadow} \mathrm{is} \mathrm{from} \mathrm{the} \mathrm{beam} \mathrm{deflector;}$ four dashed line shadows that form a square are due to a wire mesh. (b) Time-resolved pattern obtained by strobing the camera with the rotating field (integration time $\approx 5 \mathrm{~s}$ ). For a bcc $\langle 110\rangle$ crystal, a spot is predicted at each intersection of the rectangular grid lines whose separations are not adjusted.

rotating quadrupole field (rotation frequency $\left.\omega_{w}\right)$ with a potential $\propto\left(y^{2}-x^{2}\right) \cos \left(2 \omega_{w} t\right)+$ $2 x y \sin \left(2 \omega_{w} t\right)$ is generated by applying properly phased sinusoidal voltages to the 6-fold azimuthal sectors of the compensation electrodes. 3 . 9

We create ${ }^{9} \mathrm{Be}^{+}$plasmas by ionizing neutral $\mathrm{Be}$ atoms in a separate trap (not shown) and transferring the ions to the main trap for experimentation. This procedure can be repeated several times to accumulate up to $10^{6}$ ions. The trapped ${ }^{9} \mathrm{Be}^{+}$ions are then cooled to temperatures $T$ somewhat higher than the limit of $0.5 \mathrm{mK}$ by a laser beam propagating parallel to $\mathbf{B}_{0}$ at wavelength $\lambda \approx 313.11 \mathrm{~nm}^{2}$ In thermal equilibrium, the plasma takes the shape of a spheroid with uniform density and a rigid-body rotation frequency $\omega_{r}$. The density and aspect ratio of the spheroid are determined by $\omega_{r}$, for given $B_{0}$ and $V_{0}{ }^{2}$ An f $/ 5$ imaging system detects resonantly scattered photons from the axial cooling beam (diameter $\approx 0.4 \mathrm{~mm}$, power $\approx 50 \mu \mathrm{W}$ ) to produce a side-view image of the plasma, from which we measure $\omega_{r}$ (and $n_{0}$ ) with an uncertainty $5 \%$. Bragg-scattered light is detected by a CCD camera with a gateable image intensifier near the forward-scattering direction $\left(<5.4^{\circ}\right)$ since $\lambda \ll a_{\text {ws. }}{ }^{7}$ Time-resolved Bragg diffraction patterns are obtained by strobing the camera with either one of the two timing signals. ${ }^{10}$

\section{RESULTS AND DISCUSSIONS}

Figure 2(a) shows a time-averaged diffraction pattern of concentric rings from a nearly spherical plasma with $7.5 \times 10^{5}$ ions. When the pattern is time-averaged, even single crystals produce rings because of the plasma rotation about the axial laser beam. ${ }^{7}$ With the rotating field controlling the plasma rotation, we trigger the intensifier synchronously with the rotating field to open the camera for $50 \mathrm{~ns}$ each $2 \pi / \omega_{w}$ period. This enables the camera to record the diffraction pattern in the frame rotating with the quadrupole field. Figure 2(b) shows such a time-resolved pattern taken nearly simultaneously with Fig. 2(a) and accumulated over $\sim 10^{6}$ plasma rotations. The well-defined rectangular dot pattern demonstrates that the crystal is phase-locked to the rotating field with $\omega_{r}=\omega_{w} \cdot{ }^{9}$ With this phase-locked rotation, the crystalline lattice and its orientation with respect to the laser beam can last longer than $30 \min \left(\sim 10^{8}\right.$ rotations).

The diffraction pattern in Fig. 2 corresponds to a single bec crystal with a $\langle 110\rangle$ axis aligned with the laser beam. The theoretically predicted pattern agrees well with the observation within about $1 \%$. This rectangular grid pattern is essentially a plane of the reciprocal 
lattice, as can be seen from the Ewald construction in the forward-scattering limit. ${ }^{10}$ From the widths and intensities of the Bragg peaks, we estimate that the crystals consist of at least 10 lattice planes. ${ }^{7}$ Single bcc crystals with many orientations including $\langle 001\rangle,\langle 111\rangle,\langle 012\rangle$, and $\langle 115\rangle$, have also been observed, ${ }^{10}$ with $\langle 001\rangle$ and $\langle 110\rangle$ being the most frequently observed orientations.

In the future, we plan to investigate the experimental reasons for these preferred orientations. We will also attempt to directly image individual ions in crystallized plasmas with phase-locked rotation. Finally, with the improved stability of the crystal lattices obtained by the rotating field, we hope to observe the melting phase transition of the system.

We thank D. Dubin, T. O'Neil, B. King, C. Wood, M. Young, M. Lombardi, and D. Sullivan for discussions and comments. This work is supported by the Office of Naval Research.

\section{REFERENCES}

[1] J. H. Malmberg and T. M. O'Neil, Phys. Rev. Lett. 39 (1977) 1333.

[2] J. J. Bollinger, D. J. Wineland, and D. H. E. Dubin, Phys. Plasmas 1 (1994) 1403

[3] X.-P. Huang, F. Anderegg, E. M. Hollmann, C. F. Driscoll, and T. M. O'Neil, Phys. Rev. Lett. 78 (1997) 875.

[4] E. L. Pollock and J. P. Hansen, Phys. Rev. A 8 (1973) 3110 ; W. L. Slattery, G. D. Doolen, and H. E. DeWitt, ibid. 21 (1980) 2087; 26 (1982) 2255; S. Ogata and S. Ichimaru, ibid. 36 (1987) 5451; G. S. Stringfellow and H. E. DeWitt, ibid. 41 (1990) 1105; D. H. E. Dubin, ibid. 42 (1990) 4972.

[5] A. Rahman and J. P. Schiffer, Phys. Rev. Lett. 57 (1986) 1133; H. Totsuji, in Strongly Coupled Plasma Physics, ed. F. J. Rogers and H. E. DeWitt (Plenum, New York, 1987) p. 19; D. H. E. Dubin and T. M. O'Neil, Phys. Rev. Lett. 60 (1988) 511 ; J. P. Schiffer, Phys. Rev. Lett. 61 (1988) 1843; R. W. Hasse and V. V. Avilov, Phys. Rev. A 44 (1991) 4506; J. P. Schiffer, in Non-neutral Plasma Physics II, ed. J. Fajans and D. H. E. Dubin, AIP Conf. Proc. 331 (AIP Press, New York, 1995) p. 191; D. H. E. Dubin and T. M. O'Neil, in Strongly Coupled Plasma Physics, ed. S. Ichimaru (Elsevier, 1990) p. 189.

[6] S. L. Gilbert, J. J. Bollinger, and D. J. Wineland, Phys. Rev. Lett. 60 (1988) 2022; G. Birkl, S. Kassner, and H. Walther, Nature 375 (1992) 310.

[7] J. N. Tan, J. J. Bollinger, B. Jelenkovic, and D. J. Wineland, Phys. Rev. Lett. 75 (1995) 4198; J. N. Tan, J. J. Bollinger, B. Jelenkovic, W. M. Itano, and D. J. Wineland, in Physics of Strongly Coupled Plasmas, ed. W. D. Kraeft and M. Schlanges (World Scientific, Singapore, 1996) p. 387.

' [8] D. H. E. Dubin, Phys. Rev. A 40 (1989) 1140.

[9] X.-P. Huang, J. J. Bollinger, T. B. Mitchell, and W. M. Itano, Phys. Rev. Lett. 80 (1998), 73.

[10] W. M. Itano, J. J. Bollinger, J. N. Tan, B. Jelenkovic, X.-P. Huang, and D. J. Wineland,"Bragg Diffraction from Crystallized Ion Plasmas," Science (in press). 